\title{
Double Discontinuous Inverse Problems for Sturm-Liouville Operator with Parameter-Dependent Conditions
}

\author{
A. S. Ozkan, B. Keskin, and Y. Cakmak \\ Department of Mathematics, Faculty of Arts \& Science, Cumhuriyet University, 58140 Sivas, Turkey \\ Correspondence should be addressed to A. S. Ozkan; asozkan58@gmail.com
}

Received 26 March 2013; Accepted 24 June 2013

Academic Editor: Dumitru Motreanu

Copyright (C) 2013 A. S. Ozkan et al. This is an open access article distributed under the Creative Commons Attribution License, which permits unrestricted use, distribution, and reproduction in any medium, provided the original work is properly cited.

\begin{abstract}
The purpose of this paper is to solve the inverse spectral problems for Sturm-Liouville operator with boundary conditions depending on spectral parameter and double discontinuities inside the interval. It is proven that the coefficients of the problem can be uniquely determined by either Weyl function or given two different spectral sequences.
\end{abstract}

\section{Introduction}

Spectral problems of differential operators are studied in two main branches, namely, direct spectral problems and inverse spectral problems. Direct problems of spectral analysis consist in investigating the spectral properties of an operator. On the other hand, inverse problems aim at recovering operators from their spectral characteristics. Such problems often appear in mathematics, physics, mechanics, electronics, geophysics, and other branches of natural sciences.

First and most important results for inverse problem of a regular Sturm-Liouville operator were given by Ambartsumyan in 1929 [1] and Borg in 1946 [2]. Physical applications of inverse spectral problems can be found in several works (see, e.g., [3-9] and references therein).

Eigenvalue-dependent boundary conditions were studied extensively. The references $[10,11]$ are well-known examples for problems with boundary conditions that depend linearly on the eigenvalue parameter. In $[10,12]$, an operator-theoretic formulation of the problems with the spectral parameter contained in only one of the boundary conditions has been given. Inverse problems according to various spectral data for eigenparameter linearly dependent Sturm-Liouville operator were investigated in [13-17]. Boundary conditions that depend nonlinearly on the spectral parameter were also considered in [18-23].

Boundary value problems with discontinuity condition appear in the various problems of the applied sciences. These kinds of problems are well studied (see, e.g., [24-31]).
In this study, we consider a boundary value problem generated by the Sturm-Liouville equation:

$$
\ell y:=-y^{\prime \prime}+q(x) y=\lambda y, \quad x \in I=\bigcup_{i=0}^{2}\left(d_{i}, d_{i+1}\right)
$$

subject to the boundary conditions

$$
\begin{gathered}
U(y):=\lambda\left(y^{\prime}(0)+h_{0} y(0)\right)-h_{1} y^{\prime}(0)-h_{2} y(0)=0, \\
V(y):=\lambda\left(y^{\prime}(1)+H_{0} y(1)\right)-H_{1} y^{\prime}(1)-H_{2} y(1)=0
\end{gathered}
$$

and double discontinuity conditions

$$
\begin{gathered}
y\left(d_{i}+0\right)=\alpha_{i} y\left(d_{i}-0\right), \\
y^{\prime}\left(d_{i}+0\right)=\alpha_{i}^{-1} y^{\prime}\left(d_{i}-0\right)-\left(\gamma_{i} \lambda+\beta_{i}\right) y\left(d_{i}-0\right),
\end{gathered}
$$

where $q(x)$ is real valued function in $L_{2}(0,1) ; h_{j}$ and $H_{j}, j=$ $0,1,2$, are real numbers; $\alpha_{i}, \gamma_{i} \in \mathbb{R}^{+}, \beta_{i} \in \mathbb{R}, i=1,2 ; d_{0}=$ $0, d_{1}, d_{2} \in(0,1), d_{3}=1 ; \rho_{1}:=h_{2}-h_{0} h_{1}>0, \rho_{2}:=H_{0} H_{1}-$ $H_{2}>0$; and $\lambda$ is a spectral parameter. We denote the problem (1)-(4) by $L=L\left(q, \mathbf{h}, \mathbf{H}, \mathbf{s}_{1}, \mathbf{s}_{2}\right)$, where $\mathbf{h}=\left(h_{0}, h_{1}, h_{2}\right), \mathbf{H}=$ $\left(H_{0}, H_{1}, H_{2}\right)$, and $\mathbf{s}_{i}=\left(d_{i}, \alpha_{i}, \gamma_{i}, \beta_{i}\right), i=1,2$.

It is proven that the coefficients of the problem can be uniquely determined by either Weyl function or given two different spectral sequences. The obtained results are generalizations of the similar results for the classical SturmLiouville operator on a finite interval. 


\section{Preliminaries}

Let the functions $\varphi(x, \lambda)$ and $\psi(x, \lambda)$ be the solutions of (1) under the following initial conditions and the jump conditions (4):

$$
\begin{aligned}
& \left(\begin{array}{c}
\varphi \\
\varphi^{\prime}
\end{array}\right)(0, \lambda)=\left(\begin{array}{c}
-\lambda+h_{1} \\
\lambda h_{0}-h_{2}
\end{array}\right), \\
& \left(\begin{array}{c}
\psi \\
\psi^{\prime}
\end{array}\right)(1, \lambda)=\left(\begin{array}{c}
-\lambda+H_{1} \\
\lambda H_{0}-H_{2}
\end{array}\right) .
\end{aligned}
$$

These solutions are the entire functions of $\lambda$ and satisfy the relation $\psi\left(x, \lambda_{n}\right)=\boldsymbol{\beta}_{n} \varphi\left(x, \lambda_{n}\right)$ for each eigenvalue $\lambda_{n}$, where $\boldsymbol{\beta}_{n}=-\left(\psi^{\prime}\left(0, \lambda_{n}\right)+h_{0} \psi\left(0, \lambda_{n}\right)\right) / \rho_{1}$.

The following asymptotics can be obtained from the integral equations given in the appendix:

$$
\begin{aligned}
& \varphi(x, \lambda)= \begin{cases}-\lambda \cos \sqrt{\lambda} x+O(\sqrt{\lambda} \exp |\tau| x) & x<d_{1}, \\
\gamma_{1} \lambda^{2}\left[\frac{\sin \sqrt{\lambda} x}{2 \sqrt{\lambda}}-\frac{\sin \sqrt{\lambda}\left(2 d_{1}-x\right)}{2 \sqrt{\lambda}}\right]+O(\lambda \exp |\tau| x), & d_{1}<x<d_{2}, \\
\frac{\gamma_{1} \gamma_{2}}{4} \lambda^{2}\left[\cos \sqrt{\lambda} x+\cos \sqrt{\lambda}\left(2 d_{1}-x\right)-\cos \sqrt{\lambda}\left(2 d_{2}-x\right)-\cos \sqrt{\lambda}\left(2 d_{2}-2 d_{1}-x\right)\right] & x>d_{2},\end{cases} \\
& \varphi^{\prime}(x, \lambda)= \begin{cases}\lambda^{3 / 2} \sin \sqrt{\lambda} x+O(\lambda \exp |\tau| x), & x<d_{1}, \\
\frac{\gamma_{1} \lambda^{2}}{2}\left[\cos \sqrt{\lambda} x+\cos \sqrt{\lambda}\left(2 d_{1}-x\right)\right]+O\left(\lambda^{3 / 2} \exp |\tau| x\right), & d_{1}<x<d_{2}, \\
-\frac{\gamma_{1} \gamma_{2}}{4} \lambda^{5 / 2}\left\{\sin \sqrt{\lambda} x-\sin \sqrt{\lambda}\left(2 d_{1}-x\right)+\sin \sqrt{\lambda}\left(2 d_{2}-x\right)+\sin \sqrt{\lambda}\left(2 d_{2}-2 d_{1}-x\right)\right\} & x>d_{2},\end{cases}
\end{aligned}
$$

where $\tau=\operatorname{Im} \sqrt{\lambda}$.

The values of the parameter $\lambda$ for which the problem $L$ has nonzero solutions are called eigenvalues, and the corresponding nontrivial solutions are called eigenfunctions.

The characteristic function $\Delta(\lambda)$ and norming constants $\alpha_{n}$ of the problem $L$ are defined as follows:

$$
\begin{aligned}
\Delta(\lambda)= & W[\varphi, \psi] \\
= & \lambda\left(\varphi^{\prime}(1, \lambda)+H_{0} \varphi(1, \lambda)\right)-H_{1} \varphi^{\prime}(1, \lambda)-H_{2} \varphi(1, \lambda) \\
= & -\lambda\left(\psi^{\prime}(0, \lambda)+h_{0} \psi(0, \lambda)\right)+h_{1} \psi^{\prime}(0, \lambda)+h_{2} \psi(0, \lambda), \\
\boldsymbol{\alpha}_{n}:= & \int_{0}^{1} \varphi^{2}\left(x, \lambda_{n}\right) d x+\frac{1}{\rho_{1}}\left(\varphi^{\prime}\left(0, \lambda_{n}\right)+h_{0} \varphi\left(0, \lambda_{n}\right)\right)^{2} \\
& +\frac{1}{\rho_{2}}\left(\varphi^{\prime}\left(1, \lambda_{n}\right)+H_{0} \varphi\left(1, \lambda_{n}\right)\right)^{2} \\
& +\alpha_{1} \gamma_{1} \varphi^{2}\left(d_{1}-0, \lambda_{n}\right)+\alpha_{2} \gamma_{2} \varphi^{2}\left(d_{2}-0, \lambda_{n}\right) .
\end{aligned}
$$

It is obvious that $\Delta(\lambda)$ is an entire function in $\lambda$ and the zeros, namely, $\left\{\lambda_{n}\right\}$ of $\Delta(\lambda)$ coincide with the eigenvalues of the problem $L$. Now, from (6) and (8), we can write

$$
\begin{aligned}
\Delta(\lambda)=-\frac{\gamma_{1} \gamma_{2}}{4} \lambda^{4} & \\
& \times\left[\frac{\sin \sqrt{\lambda}}{\sqrt{\lambda}}-\frac{\sin \sqrt{\lambda}\left(2 d_{1}-1\right)}{\sqrt{\lambda}}+\frac{\sin \sqrt{\lambda}\left(2 d_{2}-1\right)}{\sqrt{\lambda}}\right. \\
& \left.+\frac{\sin \sqrt{\lambda}\left(2 d_{2}-2 d_{1}-1\right)}{\sqrt{\lambda}}\right]+O\left(\lambda^{3} \exp |\tau|\right) .
\end{aligned}
$$

Lemma 1. See the following.

(i) All eigenvalues of the problem $L$ are real and algebraically simple; that is, $\Delta^{\prime}\left(\lambda_{n}\right) \neq 0$.

(ii) Two eigenfunctions $\varphi\left(x, \lambda_{1}\right)$ and $\varphi\left(x, \lambda_{2}\right)$, corresponding to different eigenvalues $\lambda_{1}$ and $\lambda_{2}$, are orthogonal in the sense of

$$
\begin{aligned}
\int_{0}^{1} \varphi\left(x, \lambda_{1}\right) \varphi\left(x, \lambda_{2}\right) d x \\
+\frac{1}{\rho_{1}}\left(\varphi^{\prime}\left(0, \lambda_{1}\right)+h_{0} \varphi\left(0, \lambda_{1}\right)\right)\left(\varphi^{\prime}\left(0, \lambda_{2}\right)+h_{0} \varphi\left(0, \lambda_{2}\right)\right) \\
+\frac{1}{\rho_{2}}\left(\varphi^{\prime}\left(1, \lambda_{1}\right)+H_{0} \varphi\left(1, \lambda_{1}\right)\right)\left(\varphi^{\prime}\left(1, \lambda_{2}\right)+H_{0} \varphi\left(1, \lambda_{2}\right)\right) \\
+\alpha_{1} \gamma_{1} \varphi\left(d_{1}-0, \lambda_{1}\right) \varphi\left(d_{1}-0, \lambda_{2}\right) \\
+\alpha_{2} \gamma_{2} \varphi\left(d_{2}-0, \lambda_{1}\right) \varphi\left(d_{2}-0, \lambda_{2}\right)=0 .
\end{aligned}
$$


Proof. Consider a Hilbert Space $H=L_{2}(0,1) \oplus \mathbb{C}^{4}$, equipped with the inner product

$$
\begin{aligned}
\langle Y, Z\rangle:= & \int_{0}^{1} y(x) \overline{z(x)} d x+\frac{1}{\rho_{1}} Y_{1} \overline{Z_{1}} \\
& +\frac{1}{\rho_{2}} Y_{2} \overline{Z_{2}}+\alpha_{1} \gamma_{1} Y_{3} \overline{Z_{3}}+\alpha_{2} \gamma_{2} Y_{4} \overline{Z_{4}}
\end{aligned}
$$

for $Y=\left(y(x), Y_{1}, Y_{2}, Y_{3}, Y_{4}\right)^{T}, Z=\left(z(x), Z_{1}, Z_{2}, Z_{3}, Z_{4}\right)^{T} \in$ $H$.

Define an operator $T$ with the domain $D(T)=\{Y \in H$ : $y(x)$, and $y^{\prime}(x)$ are absolutely continuous in $I, \ell Y \in L_{2}(0$, $1), y\left(d_{i}+0\right)=\alpha_{i} y\left(d_{i}-0\right), Y_{1}=y^{\prime}(0)+h_{0} y(0), Y_{2}=y_{1}^{\prime}(1)+$ $H_{0} y(1), Y_{3}=\gamma_{1} y\left(d_{1}-0\right)$, and $\left.Y_{4}=\gamma_{2} y\left(d_{2}-0\right)\right\}$ such that

$$
T(Y):=\left(\begin{array}{c}
-y^{\prime \prime}(x)+q(x) y(x) \\
h_{1} y^{\prime}(0)+h_{2} y(0) \\
H_{1} y^{\prime}(1)+H_{2} y(1) \\
-y^{\prime}\left(d_{1}+0\right)+\alpha_{1}^{-1} y^{\prime}\left(d_{1}-0\right)-\beta_{1} y\left(d_{1}-0\right) \\
-y^{\prime}\left(d_{2}+0\right)+\alpha_{2}^{-1} y^{\prime}\left(d_{2}-0\right)-\beta_{2} y\left(d_{2}-0\right)
\end{array}\right) .
$$

It is easily proven, using classical methods in the similar works (see, e.g., [28]), that the operator $T$ is symmetric in $H$; the eigenvalue problem for the operator $T$ and the problem $L$ coincide. Therefore, all eigenvalues are real, and two different eigenfunctions are orthogonal.

Let us show the simplicity of the eigenvalues $\lambda_{n}$ by writting the following equations:

$$
\begin{gathered}
-\psi^{\prime \prime}(x, \lambda)+q(x) \psi(x, \lambda)=\lambda \psi(x, \lambda), \\
-\varphi^{\prime \prime}\left(x, \lambda_{n}\right)+q(x) \varphi\left(x, \lambda_{n}\right)=\lambda_{n} \varphi\left(x, \lambda_{n}\right) .
\end{gathered}
$$

If these equations are multiplied by $\varphi\left(x, \lambda_{n}\right)$ and $\psi(x, \lambda)$, respectively, subtracting them side by side and finally integrating over the interval $[0,1]$, the equality

$$
\begin{aligned}
& {\left[\varphi^{\prime}\left(x, \lambda_{n}\right) \psi(x, \lambda)-\psi^{\prime}(x, \lambda) \varphi\left(x, \lambda_{n}\right)\right]} \\
& \quad \times\left(\left.\right|_{0} ^{d_{1}-0}+\left.\right|_{d_{1}+0} ^{d_{2}-0}+\left.\right|_{d_{2}+0} ^{1}\right) \\
& =\left(\lambda-\lambda_{n}\right) \int_{0}^{1} \psi(x, \lambda) \varphi\left(x, \lambda_{n}\right) d x
\end{aligned}
$$

is obtained. Add and subtract $\Delta(\lambda)$ in the left-hand side of the last equality, and use initial conditions (5) to get

$$
\begin{aligned}
\Delta(\lambda) & +\left(\lambda-\lambda_{n}\right)\left(\psi^{\prime}(0, \lambda)+h_{0} \psi(0, \lambda)\right) \\
& -\left(\lambda-\lambda_{n}\right)\left(\varphi^{\prime}\left(1, \lambda_{n}\right)+H_{0} \varphi\left(1, \lambda_{n}\right)\right) \\
& -\left(\lambda-\lambda_{n}\right) \alpha_{1} \gamma_{1} \psi\left(d_{1}-0, \lambda\right) \varphi\left(d_{1}-0, \lambda_{n}\right) \\
& -\left(\lambda-\lambda_{n}\right) \alpha_{2} \gamma_{2} \psi\left(d_{2}-0, \lambda\right) \varphi\left(d_{2}-0, \lambda_{n}\right) \\
= & \left(\lambda-\lambda_{n}\right) \int_{0}^{1} \psi(x, \lambda) \varphi\left(x, \lambda_{n}\right) d x .
\end{aligned}
$$

Rewrite this equality as

$$
\begin{aligned}
& \frac{\Delta(\lambda)}{\lambda-\lambda_{n}}=\int_{0}^{1} \psi(x, \lambda) \varphi\left(x, \lambda_{n}\right) d x \\
& +\left(\varphi^{\prime}\left(1, \lambda_{n}\right)+H_{0} \varphi\left(1, \lambda_{n}\right)\right) \\
& -\left(\psi^{\prime}(0, \lambda)+h_{0} \psi(0, \lambda)\right) \\
& +\alpha_{1} \gamma_{1} \psi\left(d_{1}-0, \lambda\right) \varphi\left(d_{1}-0, \lambda_{n}\right) \\
& +\alpha_{2} \gamma_{2} \psi\left(d_{2}-0, \lambda\right) \varphi\left(d_{2}-0, \lambda_{n}\right) \\
& =\int_{0}^{1} \psi(x, \lambda) \varphi\left(x, \lambda_{n}\right) d x \\
& -\frac{\left(\psi^{\prime}(0, \lambda)+h_{0} \psi(0, \lambda)\right)\left(\varphi^{\prime}\left(0, \lambda_{n}\right)+h_{0} \varphi\left(0, \lambda_{n}\right)\right)}{h_{0} h_{1}-h_{2}} \\
& +\frac{\left(\varphi^{\prime}\left(1, \lambda_{n}\right)+H_{0} \varphi\left(1, \lambda_{n}\right)\right)\left(\psi^{\prime}(1, \lambda)+H_{0} \psi(1, \lambda)\right)}{H_{0} H_{1}-H_{2}} \\
& +\alpha_{1} \gamma_{1} \psi\left(d_{1}-0, \lambda\right) \varphi\left(d_{1}-0, \lambda_{n}\right) \\
& +\alpha_{2} \gamma_{2} \psi\left(d_{2}-0, \lambda\right) \varphi\left(d_{2}-0, \lambda_{n}\right) \\
& =\int_{0}^{1} \psi(x, \lambda) \varphi\left(x, \lambda_{n}\right) d x \\
& +\frac{\left(\psi^{\prime}(0, \lambda)+h_{0} \psi(0, \lambda)\right)\left(\varphi^{\prime}\left(0, \lambda_{n}\right)+h_{0} \varphi\left(0, \lambda_{n}\right)\right)}{\rho_{1}} \\
& +\frac{\left(\varphi^{\prime}\left(1, \lambda_{n}\right)+H_{0} \varphi\left(1, \lambda_{n}\right)\right)\left(\psi^{\prime}(1, \lambda)+H_{0} \psi(1, \lambda)\right)}{\rho_{2}} \\
& +\alpha_{1} \gamma_{1} \psi\left(d_{1}-0, \lambda\right) \varphi\left(d_{1}-0, \lambda_{n}\right) \\
& +\alpha_{2} \gamma_{2} \psi\left(d_{2}-0, \lambda\right) \varphi\left(d_{2}-0, \lambda_{n}\right) \text {. }
\end{aligned}
$$

As $\lambda \rightarrow \lambda_{n}$

$$
\Delta^{\prime}\left(\lambda_{n}\right)=\beta_{n} \boldsymbol{\alpha}_{n}
$$

is obtained by using the equality $\psi\left(x, \lambda_{n}\right)=\boldsymbol{\beta}_{n} \varphi\left(x, \lambda_{n}\right)$. Thus, $\Delta^{\prime}\left(\lambda_{n}\right) \neq 0$.

\section{Main Results}

We consider three statements of the inverse problem for the boundary value problem $L$; from the Weyl function, from the spectral data $\left\{\lambda_{n}, \boldsymbol{\alpha}_{n}\right\}_{n \geq 0}$, and from two spectra $\left\{\lambda_{n}, \mu_{n}\right\}_{n \geq 0}$. For studying the inverse problem, we consider a boundary value problem $\widetilde{L}$, together with $L$, of the same form but with different coefficients $\widetilde{q}(x), \widetilde{\mathbf{h}}, \widetilde{\mathbf{H}}, \widetilde{\mathbf{s}}_{i}, i=1,2$.

Let the function $\varkappa(x, \lambda)$ denote the solution of (1) under the initial conditions $\varkappa(0, \lambda)=\rho_{1}^{-1}, \varkappa^{\prime}(0, \lambda)=-\rho_{1}^{-1} h_{0}$ 
and the jump conditions (4). It is clear that the function $\psi(x$, $\lambda$ ) can be represented by

$$
\psi(x, \lambda)=\Delta(\lambda) \varkappa(x, \lambda)-\frac{\psi^{\prime}(0, \lambda)+h_{0} \psi(0, \lambda)}{\rho_{1}} \varphi(x, \lambda) .
$$

Denote

$$
m(\lambda):=\frac{\psi^{\prime}(0, \lambda)+h_{0} \psi(0, \lambda)}{\rho_{1} \Delta(\lambda)} .
$$

Then, we have

$$
\frac{\psi(x, \lambda)}{\Delta(\lambda)}=\varkappa(x, \lambda)-m(\lambda) \varphi(x, \lambda) .
$$

The function $m(\lambda)$ is called Weyl function [32].

Theorem 2. If $m(\lambda)=\widetilde{m}(\lambda)$, then $L=\widetilde{L}$; that is, $q(x)=\widetilde{q}(x)$, always everywhere in $I ; \mathbf{h}=\widetilde{\mathbf{h}}, \mathbf{H}=\widetilde{\mathbf{H}}$, and $\mathbf{s}_{i}=\widetilde{\mathbf{s}}_{i}, i=1,2$.

Proof. Let us define the functions $P_{1}(x, \lambda)$ and $P_{2}(x, \lambda)$ as follows:

$$
\begin{gathered}
P_{1}(x, \lambda)=\varphi(x, \lambda) \widetilde{\Phi}^{\prime}(x, \lambda)-\Phi(x, \lambda) \widetilde{\varphi}^{\prime}(x, \lambda), \\
P_{2}(x, \lambda)=\Phi(x, \lambda) \widetilde{\varphi}(x, \lambda)-\varphi(x, \lambda) \widetilde{\Phi}(x, \lambda),
\end{gathered}
$$

where $\Phi(x, \lambda)=\psi(x, \lambda) / \Delta(\lambda)$. If $m(\lambda)=\widetilde{m}(\lambda)$, then from (22)-(23), $P_{1}(x, \lambda)$ and $P_{2}(x, \lambda)$ are entire functions in $\lambda$. Denote $G_{\delta}=\left\{\lambda: \lambda=k^{2},\left|k-k_{n}\right|>\delta, n=1,2, \ldots\right\}$ and $\widetilde{G}_{\delta}=\left\{\lambda: \lambda=k^{2},\left|k-\widetilde{k}_{n}\right|>\delta, n=1,2, \ldots\right\}$, where $\delta$ is sufficiently small number and $k_{n}$ and $\tilde{k}_{n}$ are square roots of the eigenvalues of the problem $L$ and $\widetilde{L}$, respectively. One can easily show that the asymptotics

$$
\begin{aligned}
& \Phi(x, \lambda)=O\left(\lambda^{-(i+3) / 2} \exp (-|\tau| x)\right), \\
& \Phi^{\prime}(x, \lambda)=O\left(\lambda^{-(i+2) / 2} \exp (-|\tau| x)\right)
\end{aligned}
$$

are valid for $d_{i}<x<d_{i+1}, i=0,1,2$, and sufficiently large $|\lambda|$ in $G_{\delta} \cap \widetilde{G}_{\delta}$. Thus, the following inequalities are obtained from (6) and (24):

$$
\begin{array}{r}
\left|P_{1}(x, \lambda)\right| \leq C_{\delta}, \quad\left|P_{2}(x, \lambda)\right| \leq C_{\delta}|\lambda|^{-1 / 2}, \\
\lambda \in G_{\delta} \cap \widetilde{G}_{\delta} .
\end{array}
$$

According to the last inequalities and Liouville's theorem, $P_{1}(x, \lambda)=A(x)$ and $P_{2}(x, \lambda)=0$. Use (23) again to take

$$
\begin{gathered}
\varphi(x, \lambda)=A(x) \widetilde{\varphi}(x, \lambda), \\
\Phi(x, \lambda)=A(x) \widetilde{\Phi}(x, \lambda) .
\end{gathered}
$$

Since $W[\Phi(x, \lambda), \varphi(x, \lambda)]=1$ and similarly $W[\widetilde{\Phi}(x, \lambda), \widetilde{\varphi}(x$, $\lambda)]=1$, then $A^{2}(x)=1$.

On the other hand, the asymptotic expressions

$$
\begin{gathered}
\varphi(x, \lambda)=C(\lambda) \exp (-i \sqrt{\lambda} x)(1+o(1)), \\
\varphi(x, \lambda)=\widetilde{C}(\lambda) \exp (-i \sqrt{\lambda} x)(1+o(1))
\end{gathered}
$$

are valid for $\sqrt{\lambda} \rightarrow \infty$ on the imaginary axis, where

$$
\begin{gathered}
C(\lambda)= \begin{cases}-\frac{1}{2} \lambda, & 0<x<d_{1}, \\
\frac{\gamma_{1}}{4} \lambda^{3 / 2}, & d_{1}<x<d_{2}, \\
\frac{\gamma_{1} \gamma_{2}}{8} \lambda^{2}, & d_{2}<x<1,\end{cases} \\
\widetilde{C}(\lambda)= \begin{cases}-\frac{1}{2} \lambda, & 0<x<\widetilde{d}_{1}, \\
\frac{\widetilde{\gamma}_{1}}{4} \lambda^{3 / 2}, & \widetilde{d}_{1}<x<\widetilde{d}_{2}, \\
\frac{\widetilde{\gamma}_{1} \widetilde{\gamma}_{2}}{8} \lambda^{2}, & \widetilde{d}_{2}<x<1 .\end{cases}
\end{gathered}
$$

Assume that $d_{1} \neq \widetilde{d}_{1}$ and $d_{2} \neq \widetilde{d}_{2}$. There are six different cases for the permutation of the numbers $d_{i}$ and $\widetilde{d}_{i}$. Without loss of generality, let $0<d_{1}<\tilde{d}_{1}<d_{2}<\tilde{d}_{2}<1$.

From (26)-(27), we get $\gamma_{1}=\widetilde{\gamma}_{1}, \gamma_{2}=\widetilde{\gamma}_{2}$, and $A(x) \equiv 1$, while $x \in\left[0, d_{1}\right) \cup\left(\tilde{d}_{1}, d_{2}\right) \cup\left(\tilde{d}_{2}, 1\right]$.

Moreover, we get

$$
2 \lambda^{-1 / 2}(1+o(1)) A(x)+\gamma_{1}=o(1),
$$

while $x \in\left(d_{1}, \tilde{d}_{1}\right)$. By taking limit in (29) as $|\lambda| \rightarrow \infty$, we condradict $\gamma_{1}>0$. Thus, $d_{1}=\widetilde{d}_{1}$. Similarly, $d_{2}=\widetilde{d}_{2}$, and $A(x)=1$ in $I$. Hence,

$$
\varphi(x, \lambda)=\tilde{\varphi}(x, \lambda), \quad \frac{\psi^{\prime}(x, \lambda)}{\psi(x, \lambda)}=\frac{\widetilde{\psi}^{\prime}(x, \lambda)}{\widetilde{\psi}(x, \lambda)} .
$$

It can be obtained from (1), (4), and (5) that $q(x)=\widetilde{q}(x)$, a.e. in $I ; \mathbf{s}_{i}=\widetilde{\mathbf{s}}_{i}, i=1,2$, and $\mathbf{h}=\widetilde{\mathbf{h}}, \mathbf{H}=\widetilde{\mathbf{H}}$. Consequently, $L=\widetilde{L}$.

Theorem 3. If $\left\{\lambda_{n}, \boldsymbol{\alpha}_{n}\right\}_{n \geq 0}=\left\{\tilde{\lambda}_{n}, \widetilde{\boldsymbol{\alpha}}_{n}\right\}_{n \geq 0}$, then $L=\widetilde{L}$.

Proof. The meromorphic function $m(\lambda)$ has simple poles at $\lambda_{n}$, and its residues at these poles are

$$
\begin{aligned}
\operatorname{Res}\left\{m(\lambda), \lambda_{n}\right\} & =\frac{\psi^{\prime}\left(0, \lambda_{n}\right)+h_{0} \psi\left(0, \lambda_{n}\right)}{\rho_{1} \Delta^{\prime}\left(\lambda_{n}\right)} \\
& =-\frac{\boldsymbol{\beta}_{n}}{\Delta^{\prime}\left(\lambda_{n}\right)}=-\frac{1}{\boldsymbol{\alpha}_{n}} .
\end{aligned}
$$

Denote $\Gamma_{n}=\left\{\lambda:|\lambda|=\left(\sqrt{\lambda_{n}}+\varepsilon\right)^{2}\right\}$, where $\varepsilon$ is sufficiently small number. Consider the contour integral

$$
F_{n}(\lambda)=\frac{1}{2 \pi i} \int_{\Gamma_{n}} \frac{m(\eta)}{(\eta-\lambda)} d \eta, \quad \lambda \in \operatorname{int} \Gamma_{n} .
$$

There exists a constant $C_{\delta}>0$ such that $\Delta(\lambda) \geq$ $|\lambda|^{7 / 2} C_{\delta} \exp |\tau|$ holds for $\lambda \in G_{\delta}$. Use this inequality and (21) to get $|m(\lambda)| \leq C_{\delta} /|\lambda|^{3 / 2}$, for $\lambda \in G_{\delta}$. Hence, $\lim _{n \rightarrow \infty} F_{n}(\lambda)=$ 0 , and so

$$
m(\lambda)=\sum_{n=0}^{\infty} \frac{1}{\alpha_{n}\left(\lambda_{n}-\lambda\right)}
$$


is obtained from residue theorem. Consequently, if $\lambda_{n}=\tilde{\lambda}_{n}$ and $\boldsymbol{\alpha}_{n}=\widetilde{\boldsymbol{\alpha}}_{n}$ for all $n$, then from (33), $m(\lambda)=\widetilde{m}(\lambda)$. Hence, Theorem 2 yields $L=\widetilde{L}$.

We consider the boundary value problem $L_{1}$ with the condition

$$
y^{\prime}(0, \lambda)+h_{0} y(0, \lambda)=0
$$

instead of (2) in L. Let $\left\{\eta_{n}^{2}\right\}_{n \geq 0}$ be the eigenvalues of the problem $L_{1}$. It is obvious that $\eta_{n}$ are zeros of $\Delta_{1}(\eta):=$ $\psi^{\prime}(0, \eta)+h_{0} \psi(0, \eta)$.

Theorem 4. If $\left\{\lambda_{n}, \eta_{n}\right\}_{n \geq 0}=\left\{\tilde{\lambda}_{n}, \tilde{\eta}_{n}\right\}_{n \geq 0}$ and $\mathbf{h}=\widetilde{\mathbf{h}}$, then $L=$ $\widetilde{L}$.

Proof. The functions $\Delta(\lambda)$ and $\Delta_{1}(\eta)$ which are entire of order $1 / 2$ can be represented by Hadamard's factorization theorem as follows:

$$
\begin{gathered}
\Delta(\lambda)=C \prod_{n=0}^{\infty}\left(1-\frac{\lambda}{\lambda_{n}}\right), \\
\Delta_{1}(\eta)=C_{1} \prod_{n=0}^{\infty}\left(1-\frac{\eta}{\eta_{n}}\right),
\end{gathered}
$$

where $C$ and $C_{1}$ are constants which depend only on $\left\{\lambda_{n}\right\}$ and $\left\{\eta_{n}\right\}$, respectively. Therefore, $\Delta(\lambda) \equiv \widetilde{\Delta}(\lambda)$ and $\Delta_{1}(\eta) \equiv \widetilde{\Delta}_{1}(\eta)$, when $\lambda_{n}=\widetilde{\lambda}_{n}$ and $\eta_{n}=\widetilde{\eta}_{n}$ for all $n$. Thus, $\psi^{\prime}(0, \eta)+$ $h_{0} \psi(0, \eta)=\widetilde{\psi}^{\prime}(0, \eta)+h_{0} \widetilde{\psi}(0, \eta)$. Moreover, $\rho_{1}=\tilde{\rho}_{1}$ since $\mathbf{h}=\widetilde{\mathbf{h}}$. Consequently, the equality (21) yields $m(\lambda) \equiv \widetilde{m}(\lambda)$. Hence, the proof is completed by Theorem 2 .

\section{Appendix}

The solution $\varphi(x, \lambda)$ satisfies the following integral equations.

$$
\text { If } x<d_{1}
$$

$$
\begin{aligned}
\varphi(x, \lambda)= & \left(\lambda h_{0}-h_{2}\right) \frac{\sin \sqrt{\lambda} x}{\sqrt{\lambda}}+\left(h_{1}-\lambda\right) \cos \sqrt{\lambda} x \\
& +\frac{1}{\sqrt{\lambda}} \int_{0}^{x} \sin \sqrt{\lambda}(x-t) q(t) \varphi(t, \lambda) d t
\end{aligned}
$$

$$
\text { if } \begin{aligned}
d_{1}<x< & d_{2}, \\
\varphi(x, \lambda)= & \frac{\lambda h_{0}-h_{2}}{\sqrt{\lambda}} \\
& \times\left(\alpha_{1}^{+} \sin \sqrt{\lambda} x+\alpha_{1}^{-} \sin \sqrt{\lambda}\left(2 d_{1}-x\right)\right) \\
& +\left(h_{1}-\lambda\right) \\
& \times\left(\alpha_{1}^{+} \cos \sqrt{\lambda} x+\alpha_{1}^{-} \cos \sqrt{\lambda}\left(2 d_{1}-x\right)\right) \\
& +\frac{\left(\gamma_{1} \lambda+\beta_{1}\right)\left(h_{1}-\lambda\right)}{2 \sqrt{\lambda}}
\end{aligned}
$$

$$
\begin{aligned}
& \times\left(\sin \sqrt{\lambda}\left(2 d_{1}-x\right)-\sin \sqrt{\lambda} x\right) \\
& +\frac{\left(\gamma_{1} \lambda+\beta_{1}\right)\left(\lambda h_{0}-h_{2}\right)}{2 \lambda} \\
& \times\left(\cos \sqrt{\lambda} x-\cos \sqrt{\lambda}\left(2 d_{1}-x\right)\right) \\
& +\frac{\left(\gamma_{1} \lambda+\beta_{1}\right)}{2 \lambda} \\
& \times \int_{0}^{d_{1}}\left(\cos \sqrt{\lambda}(x-t)-\cos \sqrt{\lambda}\left(2 d_{1}-x-t\right)\right) \\
& \times q(t) \varphi(t, \lambda) d t \\
& +\frac{1}{\sqrt{\lambda}} \int_{0}^{d_{1}}\left(\alpha_{1}^{+} \sin \sqrt{\lambda}(x-t)\right. \\
& +\frac{1}{\sqrt{\lambda}} \int_{d_{1}}^{x} \sin \sqrt{\lambda}(x-t) q(t) \varphi(t, \lambda) d t ; \\
& \left.\quad+\alpha_{1}^{-} \sin \sqrt{\lambda}\left(2 d_{1}-x-t\right)\right)
\end{aligned}
$$

if $x>d_{2}$,

$$
\begin{gathered}
\varphi(x, \lambda) \\
=-\frac{\left(h_{1}-\lambda\right)\left(\gamma_{1} \lambda+\beta_{1}\right)}{2 \sqrt{\lambda}} \\
\times\left[\alpha_{2}^{+}\left(\sin \sqrt{\lambda} x-\sin \sqrt{\lambda}\left(2 d_{1}-x\right)\right)\right. \\
+\alpha_{2}^{-}\left(\sin \sqrt{\lambda}\left(2 d_{2}-x\right)\right. \\
\left.\left.+\sin \sqrt{\lambda}\left(2 d_{2}-2 d_{1}-x\right)\right)\right] \\
+\frac{\left(\lambda h_{0}-h_{2}\right)\left(\gamma_{1} \lambda+\beta_{1}\right)}{2 \lambda} \\
\times\left[\alpha_{2}^{+}\left(\cos \sqrt{\lambda} x-\cos \sqrt{\lambda}\left(2 d_{1}-x\right)\right)\right. \\
+\alpha_{2}^{-}\left(\cos \sqrt{\lambda}\left(2 d_{1}-x\right)\right. \\
\left.\left.+\left(h_{1}-\lambda\right) \quad \cos \sqrt{\lambda}\left(2 d_{2}-2 d_{1}-x\right)\right)\right] \\
\times\left[\alpha_{1}^{+}\left(\alpha_{2}^{+} \cos \sqrt{\lambda} x+\alpha_{2}^{-} \cos \sqrt{\lambda}\left(2 d_{2}-x\right)\right)\right. \\
+\alpha_{1}^{-}\left(\alpha_{2}^{+} \cos \sqrt{\lambda}\left(2 d_{1}-x\right)\right. \\
\left.\left.\left.+2 d_{1}-x\right)\right)\right]
\end{gathered}
$$




$$
\begin{aligned}
& +\frac{\left(\lambda h_{0}-h_{2}\right)}{\sqrt{\lambda}} \\
& \times \int_{0}^{d_{1}}\left[\cos \sqrt{\lambda}(x-t)-\cos \sqrt{\lambda}\left(2 d_{1}-x-t\right)\right. \\
& \times\left[\alpha_{1}^{+}\left(\alpha_{2}^{+} \sin \sqrt{\lambda} x+\alpha_{2}^{-} \sin \sqrt{\lambda}\left(2 d_{2}-x\right)\right)\right. \\
& +\cos \sqrt{\lambda}\left(2 d_{2}-x-t\right) \\
& \left.+\cos \sqrt{\lambda}\left(2 d_{2}-2 d_{1}-x+t\right)\right] \\
& +\alpha_{1}^{-}\left(\alpha_{2}^{+} \sin \sqrt{\lambda}\left(2 d_{1}-x\right)\right. \\
& \left.\left.-\alpha_{2}^{-} \sin \sqrt{\lambda}\left(2 d_{2}-2 d_{1}-x\right)\right)\right] \\
& \times q(t) \varphi(t, \lambda) d t-\frac{1}{2 \alpha_{2} \sqrt{\lambda}} \\
& -\frac{\left(h_{1}-\lambda\right)\left(\gamma_{1} \lambda+\beta_{1}\right)\left(\gamma_{2} \lambda+\beta_{2}\right)}{4 \lambda} \\
& \times\left[\cos \sqrt{\lambda} x+\cos \sqrt{\lambda}\left(2 d_{1}-x\right)\right. \\
& -\cos \sqrt{\lambda}\left(2 d_{2}-x\right) \\
& \left.-\cos \sqrt{\lambda}\left(2 d_{2}-2 d_{1}-x\right)\right] \\
& -\frac{\left(\lambda h_{0}-h_{2}\right)\left(\gamma_{1} \lambda+\beta_{1}\right)\left(\gamma_{2} \lambda+\beta_{2}\right)}{4 \lambda^{3 / 2}} \\
& \times\left[\sin \sqrt{\lambda} x-\sin \sqrt{\lambda}\left(2 d_{1}-x\right)\right. \\
& -\sin \sqrt{\lambda}\left(2 d_{2}-x\right) \\
& \left.+\sin \sqrt{\lambda}\left(2 d_{2}-2 d_{1}-x\right)\right] \\
& +\frac{\left(h_{1}-\lambda\right)\left(\gamma_{2} \lambda+\beta_{2}\right)}{2 \sqrt{\lambda}} \\
& \times\left[\alpha_{1}^{+}\left(\sin \sqrt{\lambda}\left(2 d_{2}-x\right)-\sin \sqrt{\lambda} x\right)\right. \\
& +\alpha_{1}^{-}\left(\sin \sqrt{\lambda}\left(2 d_{1}-x\right)\right. \\
& \left.\left.+\sin \sqrt{\lambda}\left(2 d_{2}-2 d_{1}-x\right)\right)\right] \\
& +\frac{\left(\lambda h_{0}-h_{2}\right)\left(\gamma_{2} \lambda+\beta_{2}\right)}{2 \lambda} \\
& \times\left[\alpha_{1}^{+}\left(\cos \sqrt{\lambda} x-\cos \sqrt{\lambda}\left(2 d_{2}-x\right)\right)\right. \\
& -\alpha_{1}^{-}\left(\cos \sqrt{\lambda}\left(2 d_{1}-x\right)\right. \\
& \left.\left.-\cos \sqrt{\lambda}\left(2 d_{2}-2 d_{1}-x\right)\right)\right] \\
& +\frac{\alpha_{2}}{2 \sqrt{\lambda}} \int_{0}^{d_{1}}\left[\alpha _ { 1 } ^ { + } \left(\sin \sqrt{\lambda}\left(2 d_{2}-x-t\right)\right.\right. \\
& +\sin \sqrt{\lambda}(x-t)) \\
& +\alpha_{1}^{-}\left(\sin \sqrt{\lambda}\left(2 d_{1}-x-t\right)\right. \\
& \left.\left.-\sin \sqrt{\lambda}\left(2 d_{2}-2 d_{1}-x+t\right)\right)\right] \\
& \times q(t) \varphi(t, \lambda) d t+\frac{\alpha_{2}\left(\gamma_{1} \lambda+\beta_{1}\right)}{4 \lambda} \\
& \begin{array}{c}
\times \int_{0}^{d_{2}}\left[\alpha_{1}^{+}\left(\sin \sqrt{\lambda}\left(2 d_{2}-x-t\right)-\sin \sqrt{\lambda}(x-t)\right)\right. \\
-\alpha_{1}^{-}\left(\sin \sqrt{\lambda}\left(2 d_{1}-x-t\right)\right. \\
\left.\left.\quad+\sin \sqrt{\lambda}\left(2 d_{2}-2 d_{1}-x+t\right)\right)\right]
\end{array} \\
& \times q(t) \varphi(t, \lambda) d t \\
& +\frac{\left(\gamma_{1} \lambda+\beta_{1}\right)}{4 \alpha_{2} \lambda} \\
& \times \int_{0}^{d_{2}}\left[\cos \sqrt{\lambda}(x-t)-\cos \sqrt{\lambda}\left(2 d_{1}-x-t\right)\right. \\
& -\cos \sqrt{\lambda}\left(2 d_{2}-x-t\right) \\
& \left.+\cos \sqrt{\lambda}\left(2 d_{2}-2 d_{1}-x+t\right)\right] \\
& \times q(t) \varphi(t, \lambda) d t \\
& -\frac{1}{\sqrt{\lambda}} \int_{d_{1}}^{d_{2}}\left[\alpha_{2}^{+} \sin \sqrt{\lambda}(x-t)\right. \\
& \left.+\alpha_{2}^{-} \sin \sqrt{\lambda}\left(2 d_{2}-x-t\right)\right] \\
& \times q(t) \varphi(t, \lambda) d t \\
& +\frac{\left(\gamma_{2} \lambda+\beta_{2}\right)}{2 \lambda} \\
& \times \int_{0}^{d_{1}}\left[\alpha_{1}^{+}\left(\cos \sqrt{\lambda}(x-t)-\cos \sqrt{\lambda}\left(2 d_{2}-x-t\right)\right)\right. \\
& -\alpha_{1}^{-}\left(\cos \sqrt{\lambda}\left(2 d_{1}-x-t\right)\right. \\
& \left.\left.-\cos \sqrt{\lambda}\left(2 d_{2}-2 d_{1}-x+t\right)\right)\right] \\
& \times q(t) \varphi(t, \lambda) d t \\
& -\frac{\left(\gamma_{1} \lambda+\beta_{1}\right)\left(\gamma_{2} \lambda+\beta_{2}\right)}{4 \lambda^{3 / 2}} \\
& \times \int_{0}^{d_{1}}\left[\sin \sqrt{\lambda}(x-t)-\sin \sqrt{\lambda}\left(2 d_{1}-x-t\right)\right. \\
& -\sin \sqrt{\lambda}\left(2 d_{2}-x-t\right) \\
& \left.+\sin \sqrt{\lambda}\left(2 d_{2}-2 d_{1}-x+t\right)\right] \\
& \times q(t) \varphi(t, \lambda) d t \\
& -\frac{\left(\gamma_{2} \lambda+\beta_{2}\right)}{2 \lambda}
\end{aligned}
$$




$$
\begin{aligned}
& \times \int_{d_{1}}^{d_{2}}\left[\cos \sqrt{\lambda}(x-t)-\cos \sqrt{\lambda}\left(2 d_{2}-x-t\right)\right] \\
& \quad \times q(t) \varphi(t, \lambda) d t \\
& +\frac{1}{\sqrt{\lambda}} \int_{d_{2}}^{x} \sin \sqrt{\lambda}(x-t) q(t) \varphi(t, \lambda) d t
\end{aligned}
$$

where $\alpha_{i}^{ \pm}=(1 / 2)\left(\alpha_{i} \pm 1 / \alpha_{i}\right), i=1,2$.

\section{References}

[1] V. A. Ambartsumyan, "Über eine frage der eigenwerttheorie," Zeitschrift für Physik, vol. 53, pp. 690-695, 1929.

[2] G. Borg, "Eine umkehrung der Sturm-Liouvilleschen eigenwertaufgabe bestimmung der differentialgleichung durch die eigenwerte," Acta Mathematica, vol. 78, pp. 1-96, 1946.

[3] M. Kac, "Can one hear the shape of a drum?" The American Mathematical Monthly, vol. 73, no. 4, pp. 1-23, 1966.

[4] A. V. Likov and A. Yu. Mikhailov, The Theory of Heat and Mass Transfer, Gosnergoizdat, 1963.

[5] O. N. Litvinenko and V. I. Soshnikov, The Theory of Heteregeneous Lines and Their Applications in Radio Engineering, Radio, Moscow, Russia, 1964.

[6] J. R. McLaughlin and P. L. Polyakov, "On the uniqueness of a spherically symmetric speed of sound from transmission eigenvalues," Journal of Differential Equations, vol. 107, no. 2, pp. 351382, 1994.

[7] V. P. Meschanov and A. L. Feldstein, Automatic Design of Directional Couplers, Sviaz, Moscow, Russia, 1980.

[8] A. N. Tikhonov and A. A. Samarskiǔ, Equations of Mathematical Physics, Pergamon, Oxford, UK, 1990.

[9] N. N. Voitovich, B. Z. Katsenelbaum, and A. N. Sivov, Generalized Method of Eigen-Vibration in the Theory of Diffraction, Nauka, Moscow, Russia, 1997.

[10] C. T. Fulton, "Two-point boundary value problems with eigenvalue parameter contained in the boundary conditions," Proceedings of the Royal Society of Edinburgh A, vol. 77, no. 3-4, pp. 293-308, 1977.

[11] C. T. Fulton, "Singular eigenvalue problems with eigenvalue parameter contained in the boundary conditions," Proceedings of the Royal Society of Edinburgh A, vol. 87, no. 1-2, pp. 1-34, 1980.

[12] J. Walter, "Regular eigenvalue problems with eigenvalue parameter in the boundary condition," Mathematische Zeitschrift, vol. 133, pp. 301-312, 1973.

[13] P. J. Browne and B. D. Sleeman, "A uniqueness theorem for inverse eigenparameter dependent Sturm-Liouville problems," Inverse Problems, vol. 13, no. 6, pp. 1453-1462, 1997.

[14] P. J. Browne and B. D. Sleeman, "Inverse nodal problems for Sturm-Liouville equations with eigenparameter dependent boundary conditions," Inverse Problems, vol. 12, no. 4, pp. 377381, 1996.

[15] N. Yu Kapustin and E. I. Moiseev, "Oscillation properties of solutions to a nonselfadjoint spectral problem with spectral parameter in the boundary condition," Differential Equations, vol. 35, pp. 1031-1034, 1999.

[16] Y. P. Wang, "Inverse problems for Sturm-Liouville operators with interior discontinuities and boundary conditions dependent on the spectral parameter," Mathematical Methods in the Applied Sciences, vol. 36, no. 7, pp. 857-868, 2013.
[17] C.-F. Yang and Z.-Y. Huang, "A half-inverse problem with eigenparameter dependent boundary conditions," Numerical Functional Analysis and Optimization, vol. 31, no. 4-6, pp. 754-762, 2010.

[18] P. A. Binding, P. J. Browne, and K. Seddighi, "Sturm-Liouville problems with eigenparameter dependent boundary conditions," Proceedings of the Edinburgh Mathematical Society. Series II, vol. 37, no. 1, pp. 57-72, 1994.

[19] P. A. Binding and P. J. Browne, "Oscillation theory for indefinite Sturm-Liouville problems with eigenparameter-dependent boundary conditions," Proceedings of the Royal Society of Edinburgh A, vol. 127, no. 6, pp. 1123-1136, 1997.

[20] P. A. Binding, P. J. Browne, and B. A. Watson, "Inverse spectral problems for Sturm-Liouville equations with eigenparameter dependent boundary conditions," Journal of the London Mathematical Society, vol. 62, no. 1, pp. 161-182, 2000.

[21] P. A. Binding, P. J. Browne, and B. A. Watson, "Equivalence of inverse Sturm-Liouville problems with boundary conditions rationally dependent on the eigenparameter," Journal of Mathematical Analysis and Applications, vol. 291, no. 1, pp. 246-261, 2004.

[22] R. Mennicken, H. Schmid, and A. A. Shkalikov, "On the eigenvalue accumulation of Sturm-Liouville problems depending nonlinearly on the spectral parameter," Mathematische Nachrichten, vol. 189, pp. 157-170, 1998.

[23] H. Schmid and C. Tretter, "Singular Dirac systems and SturmLiouville problems nonlinear in the spectral parameter," Journal of Differential Equations, vol. 181, no. 2, pp. 511-542, 2002.

[24] R. Kh. Amirov, A. S. Ozkan, and B. Keskin, "Inverse problems for impulsive Sturm-Liouville operator with spectral parameter linearly contained in boundary conditions," Integral Transforms and Special Functions, vol. 20, no. 7-8, pp. 607-618, 2009.

[25] G. Freiling and V. Yurko, Inverse Sturm-Liouville Problems and Their Applications, Nova Science, Huntington, NY, USA, 2001.

[26] O. H. Hald, "Discontinuous inverse eigenvalue problems," Communications on Pure and Applied Mathematics, vol. 37, no. 5, pp. 539-577, 1984.

[27] B. Keskin, A. S. Ozkan, and N. Yalçin, "Inverse spectral problems for discontinuous Sturm-Liouville operator with eigenparameter dependent boundary conditions," Communications de la Faculté des Sciences de l'Université d'Ankara. Séries A1, vol. 60, no. 1, pp. 15-25, 2011.

[28] A. S. Ozkan and B. Keskin, "Spectral problems for SturmLiouville operator with boundary and jump conditions linearly dependent on the eigenparameter," Inverse Problems in Science and Engineering, vol. 20, no. 6, pp. 799-808, 2012.

[29] A. S. Ozkan, "Inverse Sturm-Liouville problems with eigenvalue-dependent boundary and discontinuity conditions," Inverse Problems in Science and Engineering, vol. 20, no. 6, pp. 857-868, 2012.

[30] V. Yurko, "Integral transforms connected with discontinuous boundary value problems," Integral Transforms and Special Functions, vol. 10, no. 2, pp. 141-164, 2000.

[31] V. Yurko, Method of Spectral Mappings in the Inverse Problem Theory, Inverse and Ill-posed Problems Series, VSP, Utrecht, The Netherlands, 2002.

[32] H. Weyl, "Über gewöhnliche differentialgleichungen mit singularitäten und die zugehörigen entwicklungen willkürlicher funktionen," Mathematische Annalen, vol. 68, no. 2, pp. 220269,1910 . 


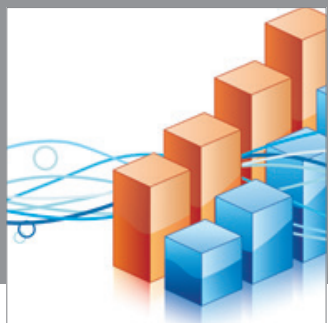

Advances in

Operations Research

mansans

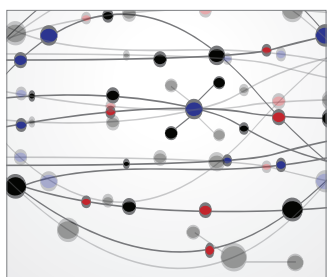

The Scientific World Journal
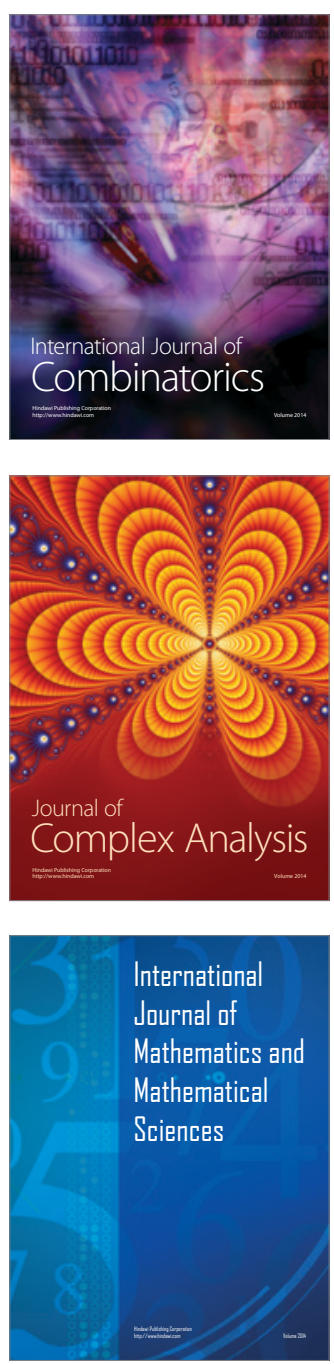
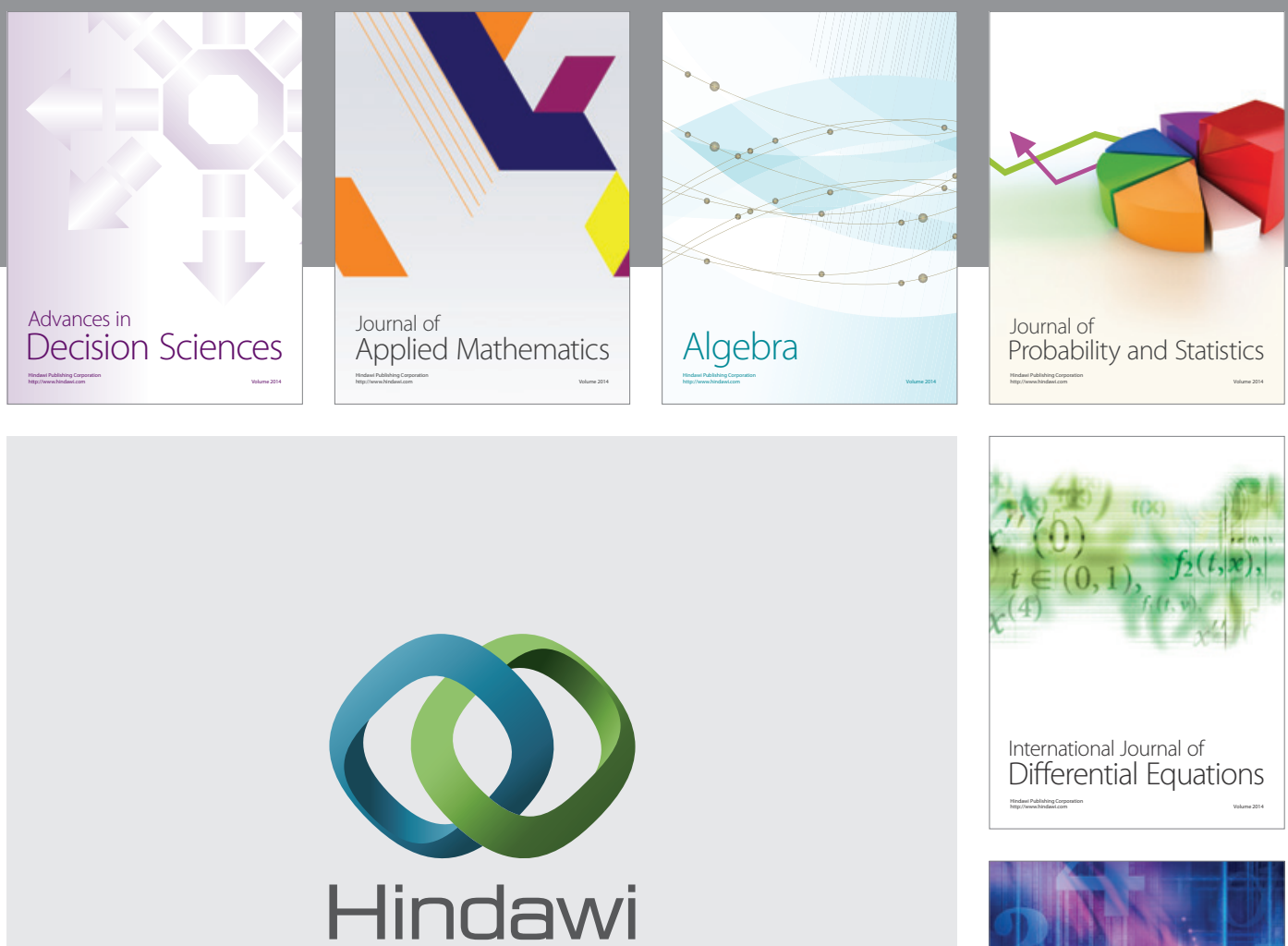

Submit your manuscripts at http://www.hindawi.com
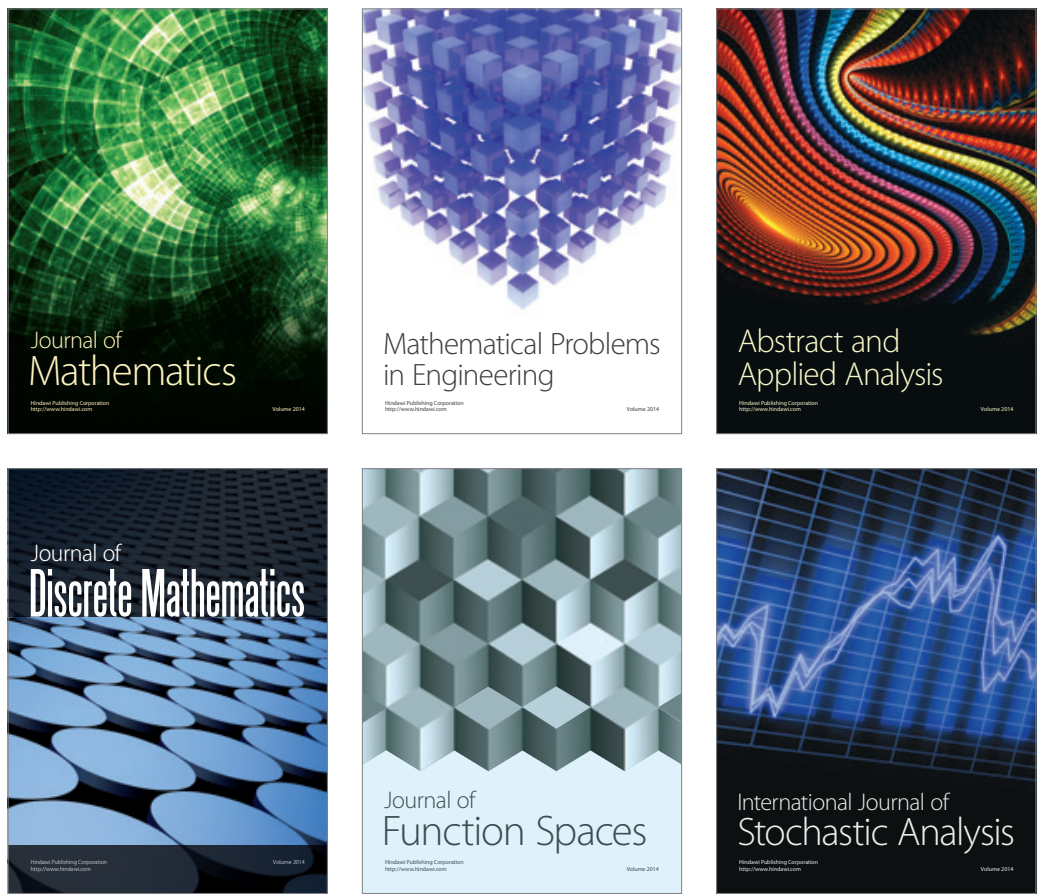

Journal of

Function Spaces

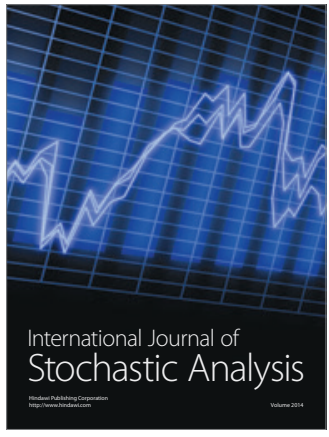

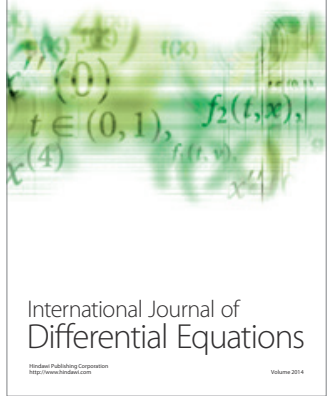
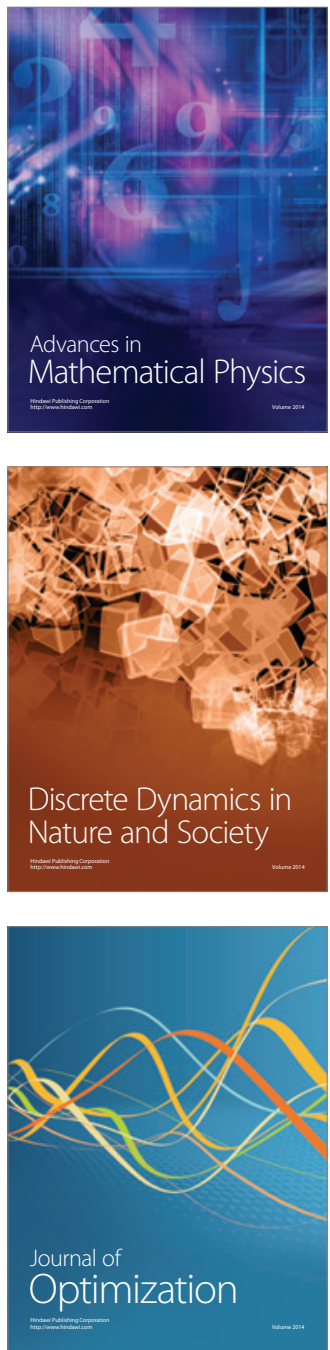